\title{
CONF-980412-
}

\section{Novel Photon Detection Based on Electronically-Induced Stress in Silicon}

\author{
P.G. Datskos ${ }^{a, b}$, S. Rajic ${ }^{a}$, I. Datskou ${ }^{c}$, C.M. Egert ${ }^{a}$ \\ a Oak Ridge National Laboratory, P.O. Box 2008, Oak Ridge, TN 37831-8039 \\ ${ }^{b}$ University of Tennessee, 401 Nielsen Physics Building, Knoxville, TN 37996-1200 \\ ${ }^{c}$ Environmental Engineering Group, 11020 Solway School Rd, Knoxville, TN 37931-2052 \\ ती 0 gिशु \\ 6
}
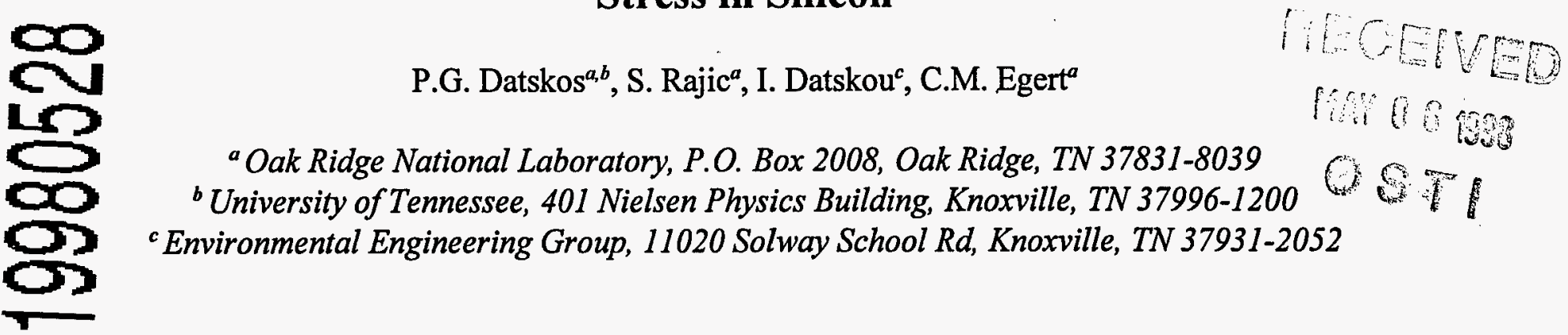

\begin{abstract}
The feasibility of microcantilever-based optical detection is demonstrated. Specifically, we report here on an evaluation of laboratory prototypes that are based on commercially available microcantilevers. In this work, optical transduction techniques were used to measure microcantilever response to photons and study the electronic stress in silicon microcantilevers, and their temporal and photometric response. The photo-generation of free charge carriers (electrons, holes) in a semiconductor gives rise to photo-induced (electronic) mechanical strain. The excess charge carriers responsible for the photo-induced stress, were produced via photon irradiation from a diode laser with wavelength $\lambda=780 \mathrm{~nm}$. We found that for silicon, the photo-induced stress results in a contraction of the crystal lattice due to the presence of excess electron-hole-pairs. In addition, the photo-induced stress is of opposite direction and about four times larger than the stress resulting from direct thermal excitation. When charge carriers are generated in a short time, a very rapid deflection of the microcantilever is observed (response time $\sim \mu \mathrm{s}$ ).
\end{abstract}

Keywords: microcantilever, photon detector, thermal detector, photo-induced stress, electron-hole pairs

\section{INTRODUCTION}

Photon detection and imaging has extensive medical, industrial, military, and commercial applications. Presently, there are several families of commercially available photon detectors, including various solid state photon detectors [1]. Photon detectors [2,3] can be classified broadly as either quantum (photon) detectors [4] or thermal detectors such as pyroelectric [5], thermoelectric, resistive microbolometers $[6,7]$ and microcantilever thermal detectors [8-10]. In photon detectors, incoming radiation is converted into electronic excitation; in thermal detectors, conversion of radiation into heat takes place which is subsequently sensed as changes in the detector temperature. Among the various of electromagnetic radiation detectors, the class photon detectors have fast response times and high detectivities, $D^{*}$. Thermal detectors have a very broadband response, since they are based upon thermal conversion of the absorbed energy. More recently, a new type of thermal detector based on microcantilevers was developed $[9,10]$ with a reported $D^{*} \sim 10^{8} \mathrm{~cm} \mathrm{~Hz}^{1 / 2} \mathrm{~W}^{-1}[9]$.

A new approach for producing compact, light-weight, highly-sensitive micromechanical photon detectors is provided by microcantilever technology, which functions based on the bending of silicon microcantilevers upon absorption of photons. When a Si microcantilever is exposed to photons (with energies above the bandgap energy), the excess charge carriers generated induce an electronic stress which causes the silicon microcantilever to deflect. This response is depicted schematically in Figure 1 . Surface stresses $s_{1}$ and $s_{2}$ are balanced at equilibrium, generating a radial force $F_{\mathrm{r}}$ along the medial plane of the microcantilever. These stresses become unequal upon exposure to photons, producing in a bending force, $F_{2}$, that displaces the tip of the microcantilever. Furthermore, if these microcantilevers are coated with a material exhibiting dissimilar thermal expansion properties than Si, the bimaterial effect will cause the

Further author information -

P.G.D. (Correspondence): E-mail: datskos@utk.edu; Telephone: (423) 574-6205; Fax: (423) 574-9407,

S.R.: E-mail: urv@ornl.gov; Telephone:(423) 574-6205; Fax: (423) 574-9407,

I.D.: Email: irene_datskou@eeg-inc.com; Telephone:(423) 927-3717; Fax: (423) 927-3817,

C.M.E.: Email: cme@ornl.gov; Telephone:(423) 574-9410; Fax: (423) 574-9407

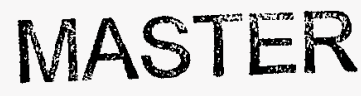




\section{DISCLAIMER}

This report was prepared as an account of work sponsored by an agency of the United States Government. Neither the United States Government nor any agency thereof, nor any of their employees, makes any warranty, express or implied, or assumes any legal liability or responsibility for the accuracy, completeness, or usefulness of any information, apparatus, product, or process disclosed, or represents that its use would not infringe privately owned rights. Reference herein to any specific commercial product, process, or service by trade name, trademark, manufacturer, or otherwise does not necessarily constitute or imply its endorsement, recommendation, or favoring by the United States Government or any agency thereof. The views and opinions of authors expressed herein do not necessarily state or reflect those of the United States Government or any agency thereof. 
$\mathrm{Si}$ microcantilever to bend even more in response to the electronic stress. The extent of bending is directly proportional to the radiation intensity.

Previous work has shown that microcantilever bending can readily be determined by a number of means, including optical, capacitive, piezoresistive, and electron tunneling with extremely high sensitivity [11]. For example, the metal-coated microcantilevers that are commonly employed in atomic force microscopy (AFM) allow sub-Angstrom $\left(<10^{-10}\right.$ meter) sensitivity to be routinely obtained. For example Hansma [12]] and Binnig [13] have demonstrated AFM sensitivities of $10^{-11}$ $\mathrm{N}$, corresponding to bending magnitudes of approximately $5 \times 10^{-11} \mathrm{~m}$. More recently, even smaller microcantilever deflections were measured with a resolution of $\sim 0.4 \times 10^{-12} \mathrm{~m}$ $[14,15]$. Standard AFM microcantilevers are typically $100-$ $200 \mu \mathrm{m}$ long, 0.3-3 $\mu \mathrm{m}$ thick and 10-30 $\mu \mathrm{m}$ wide, and can be fabricated from various dielectric or semiconducting materials. Microcantilevers made out of GaAs were also fabricated with a thickness of merely $100 \mathrm{~nm}[16]$. When even thinner microcantilevers were used, measurements of $10^{-18} \mathrm{~N}$ have been
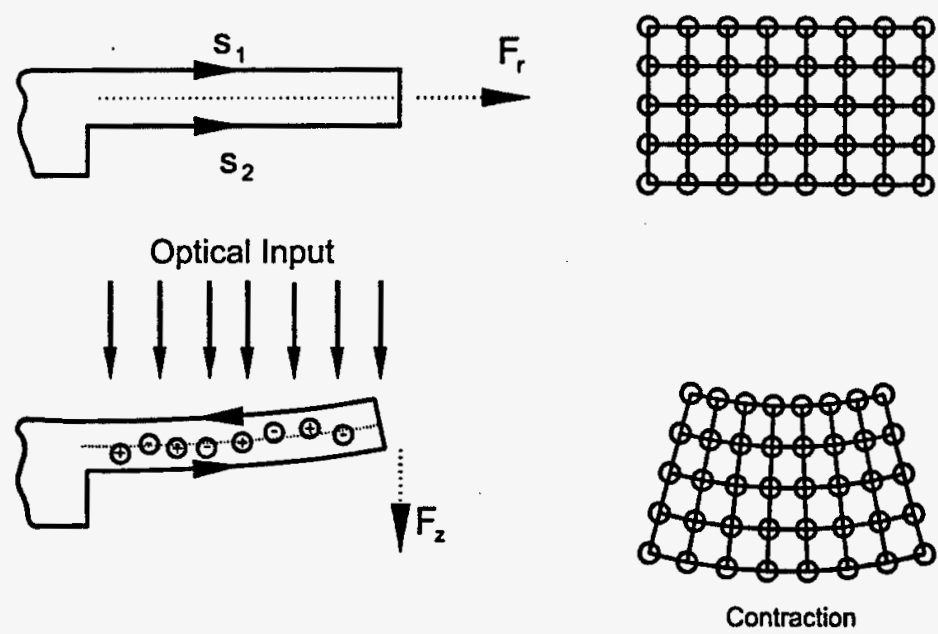

Figure 1. Schematic diagram showing the bending process of a $\mathrm{Si}$ microcantilever exposed to photons. Surface stresses $s_{1}$ and $s_{2}$ are balanced at equilibrium. Also depicted is the accompanied contraction of the silicon lattice following the generation of electron hole pairs.

reported. Microcantilevers can be mass produced at relatively low cost using standard semiconductor manufacturing methods. When microcantilevers are used as photon detectors and not as thermal detectors, they have faster response times and higher performance than that of microcantilever thermal detectors. Since microcantilevers can be easily manufactured in one- and two-dimensional arrays having 500 or more individual microcantilevers on a single wafer, this technology may be practical for manufacturing sensitive photon detector arrays with spatial resolutions comparable to current $\mathrm{CCD}$ detectors.

\section{THEORY OF ELECTRONICALLY-INDUCED STRESS}

Microcantilevers (see Figure 1) undergo bending due to the differential surface stress [17] $\left(\Delta s=s_{1}-s_{2}\right)$ created by transient expansions or contarctions; the top (photon exposed) side contracts (expands) sooner that the bottom (unexposed) side thus creating a differential surface stress. Earlier work has shown that the absorption of photons by a solid results in temperature changes and thermal expansion which in turn gives rise to acoustic waves at frequencies corresponding to the amplitude modulation of the incident photon beam [18,19]. It was also demonstrated that the elastic wave stress amplitude can be larger than the radiation pressure amplitude [18]. Acoustic signals that result from thermoelastic coupling have been used to obtain photoacoustic images of thin metallic films [19]. It has been reported earlier that photoacoustic generation in semiconductors is due primarily to photogenerated charge carriers [20] and not to thermoelastic effects [21]; of course the energy of the incident photons has to be larger than the band gap energy of the semiconductor. It is well known that in a semiconductor the generation of "free" charge carriers (electrons and holes) via photon irradiation results in the development of a local mechanical strain [20,22]. This additional strain adds to other strains resulting from temperature changes of the semiconductor. When the photon flux is high enough to cause the semiconductor to heat, the subsequent expansion (or contraction) can be detected as acoustic waves with conventional photoacoustic techniques $[19,23]$.

In a semiconductor structure of thickness $t$ and energy bandgap $\epsilon_{\mathrm{g}}$, the change in total surface stress due to photogenerated excess charge carriers, $\Delta n$, and changes in temperature, $\Delta T$, will be the sum of the additional photo-induced stress, $\Delta s_{p i}$, and thermal stress, $\Delta s_{t h}$, viz. $[20,22,24]$

$$
\Delta s=\Delta s_{p i}+\Delta s_{t h}=\left(\frac{1}{3} \frac{d \varepsilon_{g}}{d P} \Delta n\right) E+\alpha \Delta T E
$$


where, $d \epsilon_{g} / d P$ is the pressure dependence of the energy bandgap, $\alpha$ is the coefficient of thermal expansion, and $E$ is the Young's modulus. A hole (in the valence band) decreases the energy of covalent bonds while an electron adds to the bonding (or antibonding) energy. Therefore there is a competing effect between the thermal and the photo-induced stress. When $\mathrm{d} \epsilon_{g} / \mathrm{d} P$ is negative the photo-induced stress is of opposite sign than that of the thermal stress and will tend to make the semiconductor crystal to contract.

For a rectangular bar (Figure 1) of length $l$, width $\mathrm{w}$, and thickness $t$, the reciprocal of the radius of curvature, $R$, is given by Stoney's relationship [25]

$$
\frac{1}{R}=\frac{6(1-v)}{E t}\left(\Delta s_{p i}+\Delta s_{t h}\right)
$$

where, $v$ is the Poisson's ratio. Using Eqn (1) the above equation can be rewritten as [24]

$$
\frac{1}{R} \approx \frac{2(1-v)}{t} \frac{d \varepsilon_{g}}{d P} \Delta n+\frac{6(1-v)}{t} \alpha \Delta T
$$

The reciprocal of the radius of curvature is approximately equal to $d z / d y^{2}$ [26]. Then the maximum displacement $z_{\max }$ of the microcantilever is given by

$$
z_{\max } \approx \frac{(1-v) l^{2}}{t} \frac{d \varepsilon_{g}}{d P} \Delta n+\frac{3(1-v) l^{2}}{t} \alpha \Delta T
$$

The first term inside of Eqn (4) is due to photo-induced surface stress and the second term is due to thermal stress caused by temperature changes. Of course, the overall change in $z_{\max }$ will depend on several physical and mechanical properties of the semiconductor. In fact, for the same input power an Si microcantilever exhibits a photo-induced bending that is about four times larger than that due to thermal induced stress [24]. Neglecting any temperature effects that are only important in relative long time scales (comparable to the thermal time constant $>\mathrm{ms}$ ), $z_{\max }$ can be written as

$$
z_{\max } \approx \frac{(1-v) l^{2}}{t} \frac{d \varepsilon_{g}}{d P} \Delta n
$$

Assuming that an incident radiant power, $\Phi_{e}$, in a semiconductor microcantilever generates number density of excess charge carriers, $\Delta n$, we get

$$
\Delta n=\eta \frac{\lambda}{h c} \frac{\tau_{l}}{l w t} \Phi_{e}^{a b s}
$$

where $\eta$ is the quantum efficiency, $h\left(=6.625 \times 10^{-34} \mathrm{~J} \mathrm{~s}\right)$ is Planck's constant, $c\left(=3 \times 10^{8} \mathrm{~m} \mathrm{~s}^{-1}\right)$ is the speed of light, and $\tau_{L}$ is the lifetime of the carriers in the semiconductor. Then the maximum displacement $z_{\max }$ can be rewritten as 


$$
z_{\max } \approx \frac{(1-v) l^{2}}{t}\left(\eta \frac{\lambda}{h c} \frac{d \varepsilon_{g}}{d P} \frac{1}{l w t}+\frac{3 \alpha}{m c_{p}}\right) \tau_{L} \Phi_{e}
$$

We can define the deflection responsivity $\Re=z / \Phi_{e}$, viz.,

$$
\Re=\frac{(1-u) l}{w t^{2}} \frac{d \varepsilon_{g}}{d P} \frac{\lambda}{h c} \eta \tau_{L}
$$

Since the charge carriers can be generated in a very short time the photo-induced stress can manifest itself much faster than thermal stress. Of course, the overall change in $z_{\max }$ will depend on the physical and mechanical properties of the semiconductor. In fact, for the same input power a Si microcantilever exhibits a photo-induced bending that is about 4 times larger than that due to thermally-induced stress.

In Figure 2 we calculated the photo-induced deflection of a Si microcantilever with length $l=100$ $\mu \mathrm{m}$, width $w=20 \mu \mathrm{m}$, and thickness $t=0.5 \mu \mathrm{m}$ as a function of photon wavelength for an absorbed power of $1 \mathrm{nW}$. The bending due to photo-generated charges increases linearly with increasing wavelength up to the cutoff $\lambda_{c}=1.1 \mu \mathrm{m}$.

The deflection sensitivity can be further increased by coating the microcantilever with a layer of another material. It is well known that a bimaterial structure can exhibit enhanced bending sensitivity when stressed. Such a bimaterial structure will exhibit an enhanced bending for the same input power depending on the materials used. For example, the bending, $z_{\max }$, of bimaterial microcantilever can be written as [27]

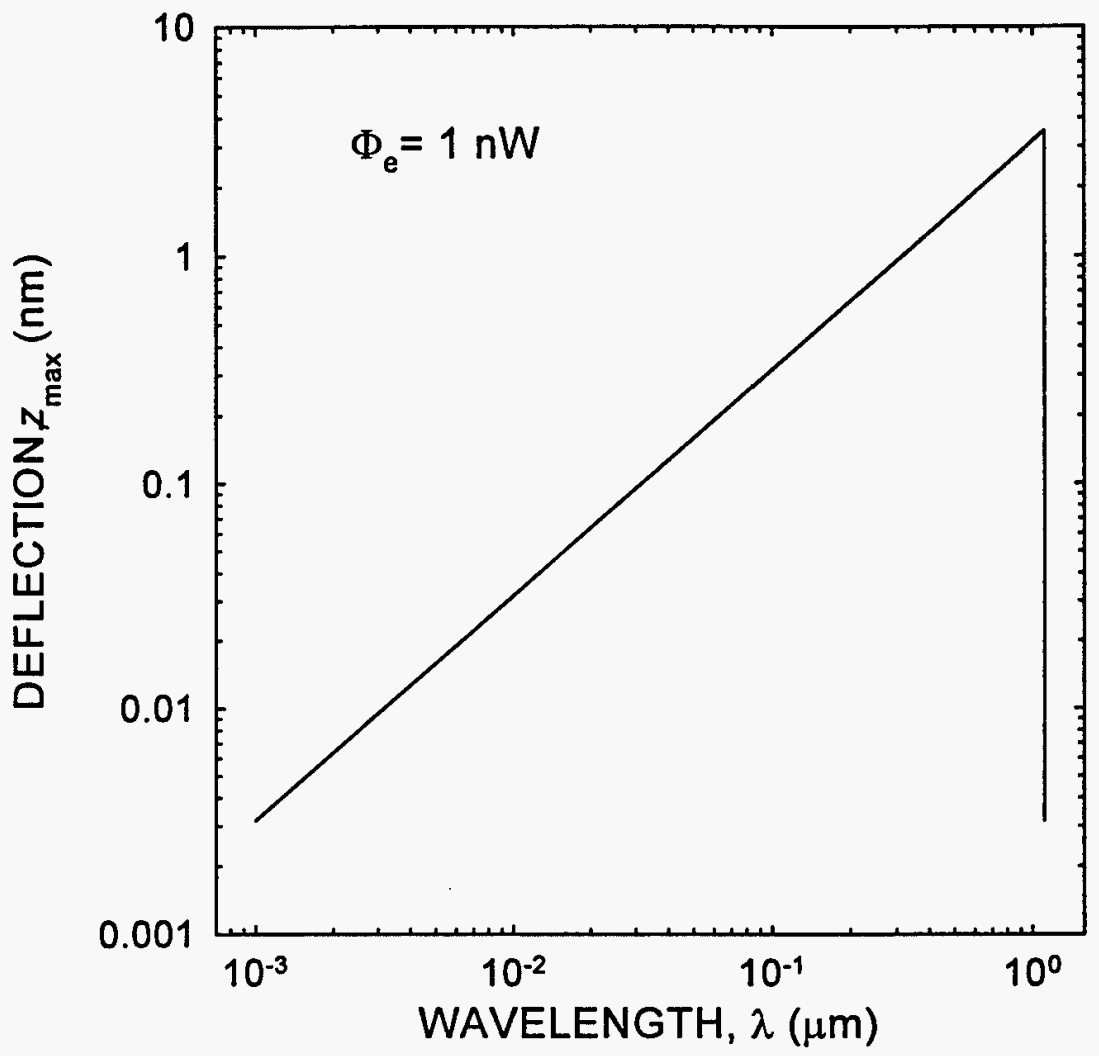

Figure 2. Calculated deflection of a Si microcantilever due to photo-induced stress as a function of photon wavelength for an absorbed power of $1 \mathrm{nW}$.

$$
z_{\max }=\frac{l^{2}}{t_{1}+t_{2}}\left(\frac{1+\left(t_{1} / t_{2}\right)^{2}}{3\left(1+t_{1} / t_{2}\right)^{2}+\left(1+t_{1} E_{1} / t_{2} E_{2}\right)\left(t_{1}^{2} / t_{2}^{2}+t_{2} E_{2} / t_{1} E_{1}\right)}\right) \frac{E_{1}}{E^{*}} \frac{d \varepsilon_{g}}{d P} \Delta n
$$

where $t_{1}$ and $t_{2}$ are the thickness of the bimaterial layer and microcantilever substrate, $l$ is the microcantilever length, $E_{1}$ and $E_{2}$ are the Young's moduli of the bimaterial layer and microcantilever, and $E^{*}$ is the effective Young's modulus of the coated microcantilever and is given by $E^{*}=E_{1} E_{2} /\left(E_{1}+E_{2}\right)$. Materials with large differences in their $E$ will offer better deflection sensitivity. However, the larger the difference between the Young's modulus the more difficult it becomes to deposit a bimaterial layer and not produce "curled" microcantilevers $[10,15]$. The deposition of metal layers on thin microcantilevers to produce unstressed structures with no bending is 
difficult and requires extremely high thermal stability. Bimaterial microcantilevers with no noticeable bending have been produced when care was taken to avoid any temperature rises during the bimaterial deposition process [15]. In those studies the investigators broke down the complete deposition process into 20 steps in order to avoid the temperature of the microcantilever rising during the deposition.

\section{EXPERIMENTAL}

Although bending of microcantilevers can readily be determined by a number of means (optical, capacitive, electron tunneling, and piezoresistive methods) in this work, we concentrated on optical readout techniques. The approach used was adapted from standard atomic force microscopy imaging systems, and is shown in Figure 3. Microcantilevers were mounted in a chip holder which secured the base of the microcantilever against a small piezoelectric transducer; the piezoelectric transducer allowed us to excite the microcantilever with different acoustic frequencies and determine its resonance frequency. The chip holder was then mounted on a three-axis translation stage to facilitate fine adjustment of the microcantilever relative to the rest of the experimental apparatus. Collimated optical radiation from a diode laser was used to evenly illuminate the mounted microcantilever (pump wavelength of $780 \mathrm{~nm}$, centered on the tip of the microcantilever $100 \mu \mathrm{m}$ in length). A mechanical chopper was used to modulate the incoming photon radiation. This configuration provided a flexible, easily controlled test system for quantifying microcantilever response to optical energy. All measurements were conducted at ambient temperature and atmospheric conditions.

A second laser was used in a probe configuration to monitor bending. A helium-neon laser (or HeNe, delivering $3 \mathrm{~mW}$ at $633 \mathrm{~nm}$ ) was focused onto the tip of the microcantilever using a $10 \times$ microscope objective; to minimize heating of the tip by the probe laser, optical power was reduced by placing a neutral density filter with an optical density of 1.0 between the probe laser and the objective. A quadelement $(\mathrm{A}, \mathrm{B}, \mathrm{C}, \mathrm{D})$ photodiode detector was used to collect the reflected probe beam. The current output $\left(i_{\mathrm{A}, \mathrm{B}, \mathrm{C}, \mathrm{D}}\right)$ of the photodiode depends linearly on the bending of the microcantilever. A high narrow bandpass optical filter is placed in front of the photodiode allowing the laser beam to be detected while preventing other wavelengths from reaching the photodiode. The amplified differential current signal from the quad cell photodiode, $i_{\mathrm{A}, \mathrm{B}, \mathrm{C}, \mathrm{D}}\left[=\left(i_{\mathrm{A}}+i_{\mathrm{B}}\right)-\left(i_{\mathrm{C}}+i_{\mathrm{D}}\right) /\left(i_{\mathrm{A}}+i_{\mathrm{B}}+i_{\mathrm{C}}+i_{\mathrm{D}}\right)\right]$, is monitored and recorded using a digital oscilloscope (TDS 780, Tektronix) or sent to a lock-in amplifier (SR850, Stanford Research Systems) for signal extraction and averaging. Optical response characteristics of a $0.6 \mu \mathrm{m}$ thick Si microcantilever was evaluated.

During these studies we also coated $\mathrm{Si}$ microcantilevers with a thin layer of $\mathrm{Al}(30 \mathrm{~nm})$. We used a broad argon ion beam and an $\mathrm{Al}$ target to sputter a thin coating on one surface of the microcantilever; we used Al coated microcantilevers to study the effect of thermal stress using a diode laser with $1300 \mathrm{~nm}$ photons. We found that even at those small thicknesses, Al causes the microcantilevers to "curl". We heated the coated microcantilevers to about $500^{\circ} \mathrm{C}$ for a period of four to six hours to reduce residual stresses. This procedure seemed to result in microcantilever structure with almost no residual bending. However, the resonance frequency of the coated microstructures was lower than the uncoated and it is rather difficult to determine how much of the shift was due to mass loading and how much due to residual stresses.

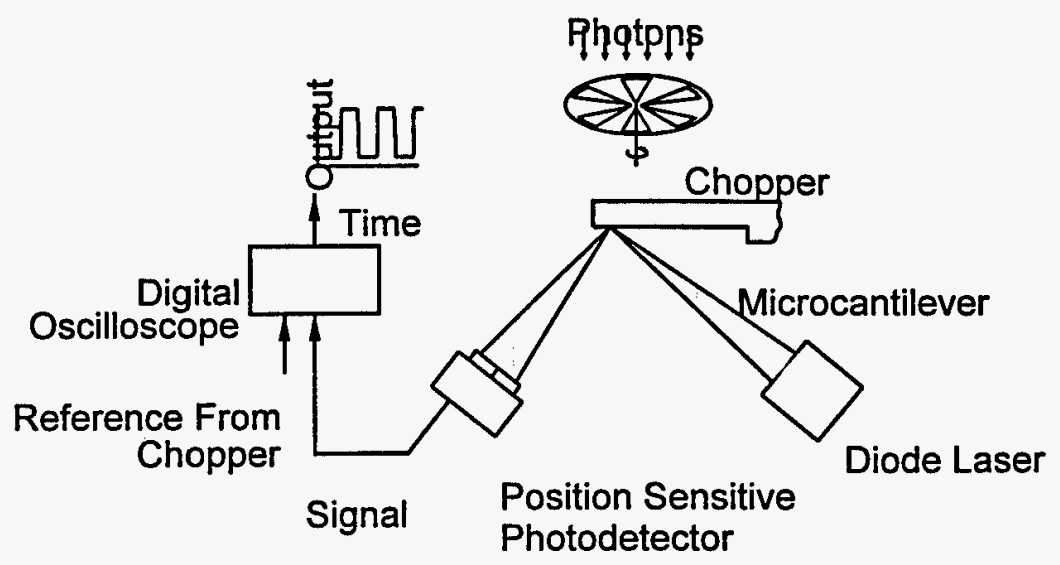

Figure 3. Schematic diagram of the experimental setup used in the present studies..

\section{RESULTS}

The microcantilever was exposed to photons from a diode laser with wavelength $\lambda=780 \mathrm{~nm}$ and using a mechanical chopper, the incoming photon radiation was modulated at a frequency of $1000 \mathrm{~Hz}$. We measured the microcantilever deflection as a function of time and and in Figure 4 we plotted that temporal response when the absorbed optical power was $3.9 \mathrm{nW}$; the absorbed power was calculated 
using $\Phi_{e}^{a b s}=\alpha_{\text {abs }} \Phi_{\underline{e}}^{\text {inc }} A_{\text {cant }} / A_{\text {spot, }}$, where $\alpha_{\text {abs }}(\approx 0.95)$ is the absorptivity of Si at $780 \mathrm{~nm}, A_{\text {cant }}$ is the cantilever area and $A_{\text {spot }}\left(=1.53 \mathrm{~mm}^{2}\right)$ is the area of the focused laser beam at the plane of the microcantilever. The observed bending is attributed to the fact that the irradiated (top) side of the microcantilever contracts sooner that the bottom side. As can be seen from Figure 4, the Si microcantilever responds rapidly to incoming photons that generate charge carriers which, in turn, cause a measurable mechanical bending. In these experiments, photons continued to impinge on the detector surface for $\sim 5 \times 10^{-4} \mathrm{~s}$, while the bending reached its maximum value in $\sim 1 \times 10^{-4} \mathrm{~s}$. The time that the microcantilever reached its maximum bending corresponds nicely to the lifetime of photo-generated "free" charge carriers in $\mathrm{Si}$. For a similar structure, thermal effects have been found to play a role in a slower time scale and have a time constant $>10^{-3} \mathrm{~s}[9,28]$.

In order to determine the effect of the thermal stress under our experimental conditions, we illuminated the Si microcantilever using photons with $\lambda=1300 \mathrm{~nm}$. Since $\mathrm{Si}$ is transparent to these photons, we deposited a thin aluminum coating of $30 \mathrm{~nm}$ along one side of the Si microcantilever using the procedure described in above. The absorbed power was estimated using $\Phi_{e}^{a b s}=\alpha_{a b s} \Phi_{e}^{i m c} A_{A l} / A_{\text {spot }}$ where $\alpha_{\mathrm{abs}}=0.01$ is the absorbivity of $\mathrm{Al}$ and $A_{\mathrm{Al}}\left(=2 \times 10^{-5} \mathrm{~cm}^{2}\right)$ is the area of the Al coating. We modulated the infrared radiation at a frequency of $1000 \mathrm{~Hz}$ and in Figure 4 curve (c) we plotted the measured microcantilever bending as a function of time for an absorbed power of 2 $\mathrm{nW}$. The change in the direction of microcantilever bending compared to curve (a), is due to the fact that the irradiated (top) side of the microcantilever expands sooner that the bottom side. From curve (c) in Figure 4 it can be seen that for $780 \mathrm{~nm}$ photons and $1000 \mathrm{~Hz}$ modulation frequency, the observed thermally induced bending of the microcantilever is smaller (over 2 times after correcting for the absorbed power) compared to the photo-induced bending and the response time is longer (>ms). The response of the microcantilever [curve (a) in Figure 4] is faster than deflections due to thermal effects [curve (c) in Figure 4].

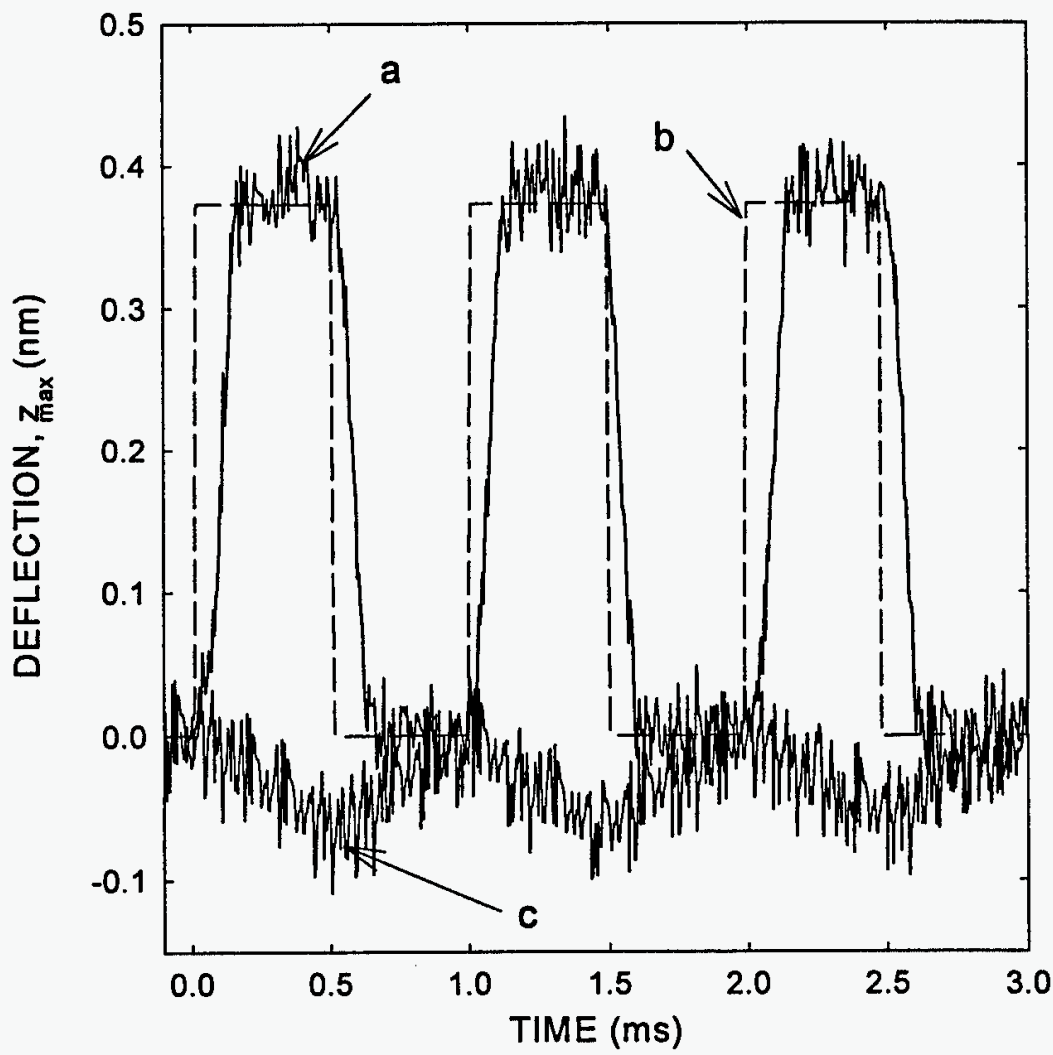

Figure 4. Deflection of a Si microcantilever [curve (a)] due to photo-generated charge carrier when exposed to photons with $\lambda=780 \mathrm{~nm}$ and an absorbed power of $3.9 \mathrm{nW}$. The dashed curve (b) represents the signal from the modulator and shows the amount of time the detector was exposed to photons. Curve (c) corresponds to deflection of the microcantilever due to absorption of $2 \mathrm{nW}$ power of photons with $\lambda=1300 \mathrm{~nm}$ and is purely due to thermal effects (see text). 


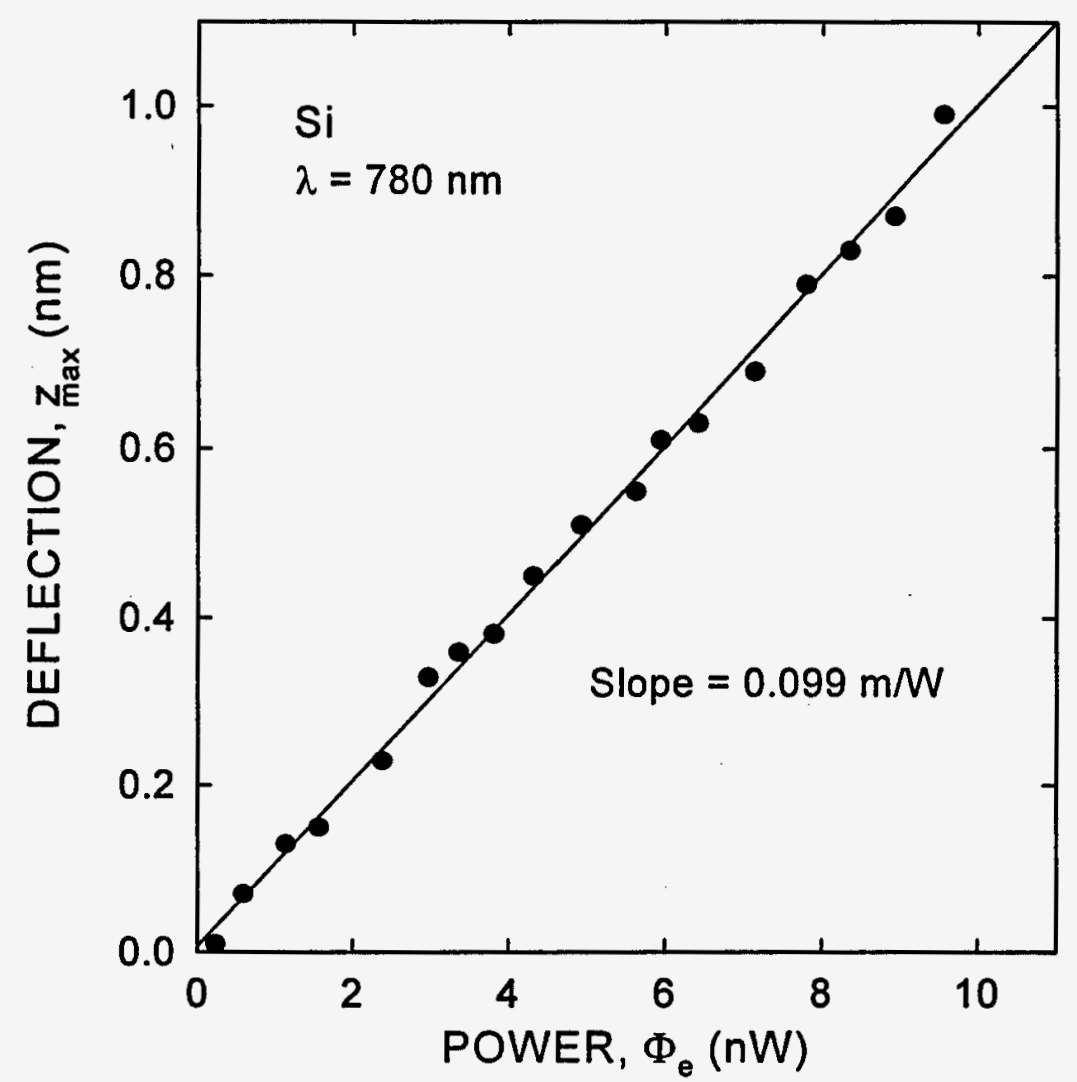

Figure 5. Deflection of a Si microcantilever as a function of absorbed power due to photo-generated charge carrier when exposed to photons with $\lambda=780 \mathrm{~nm}$.

Since $d E_{g} d P$ is negative for $\mathrm{Si}$ [29], it should be straightforward to distinguish the photo-induced stress from the thermal stress in Si by observing the direction of bending indicated by the phase shift of the signal waveform with respect to the reference signal. When photons with energies above the bandgap are used, the Si microcantilever contracts and deflects in one direction [curve (a) in Figure 4]. However, when photons with energies below the bandgap are used, the Si beam expands and deflects in the opposite direction [curve (c) in Figure 4]. We also measured the microcantilever bending due to photo-induced stress as a function of absorbed power. In Figure 5 we plotted the measured bending of a Si microcantilever as a function of absorbed power using a diode laser with $\lambda=780 \mathrm{~nm}$. The microcantilever deflection was primarily due to photo-induced stress and was found to increase linearly with increasing power with a deflection sensitivity $\Re=0.099 \mathrm{~m} / \mathrm{W}$.

\section{DISCUSSION}

An essential aspect of any scheme for micromechanical photon detection is the ability to sensitively detect physical changes resulting from photo-induced stress, since this directly affects the sensitivity and precision in measurement of photon flux. As an initial evaluation of the ability to detect optically-induced bending of a microcantilever, microcantilevers were subjected to both mechanical and optical excitation, and their response measured as a function of excitation frequency. Mechanical excitation was achieved by driving the piezoelectric element in the AFM chip holder with the reference signal from the lock-in amplifier; such a mechanical excitation response is helpful in locating resonance frequencies for allowed microcantilever bending modes.

It can be seen that the calculated deflection sensitivity for $780 \mathrm{~nm}$ photons is $\Re \sim 2.5 \mathrm{~m} / \mathrm{W}$ (see Figure 2 ) and is larger than our measured $\Re=0.099 \mathrm{~m} / \mathrm{W}$. The main reason for the observed difference is the approximation used to determine the photo-induced stress 
(Eqn 1) and possibly to the value used for the absorbivity of Si. In these studies the smallest microcantilever deflection we measured was $10^{-11} \mathrm{~m}$. However, values of $10^{-12} \mathrm{~m}$ are possible corresponding to a minimum detectable power of $10^{-11} \mathrm{~W}$ which corresponds to detectivity of $D^{*} \sim 10^{9} \mathrm{~cm} \mathrm{~Hz}^{1 / 2} \mathrm{~W}^{-1}$ at $30 \mathrm{~Hz}$. This value is one order of magnitude higher than the $D^{*}$ reported for microcantilever thermal detectors [9].

Our results demonstrate that exposing Si microcantilevers to photons with energies above the bandgap of $\mathrm{Si}$, produces a photo-induced mechanical stress which dominates over thermally-induced stresses. However, when the photon energy is below the Si bandgap thermal effects dominate. We found that the deflection of a Si microcantilever depends linearly on photo-induced stress which, in turn, depends linearly on the input optical power and is manifested with fast response times. Furthermore, this mechanism does not rely on changes in the temperature of the microstructure and therefore thermal isolation (that is crucial to the operation of thermal detectors) has minimal influence. Utilizing such a detection mechanism it may be possible to construct micromechanical photon detectors and unlike thermal detectors that respond slowly (response times $\sim \mathrm{ms}$ ) to impinging photons via temperature changes, a detector based on the photo-induced stress will respond both more sensitively and rapidly to incoming photons, with fast response times $<\mathrm{ms}$.

\section{CONCLUSIONS}

The results of the present work demonstrate that microcantilevers represent an important development in micromechanical photon detector technology, and can be expected to provide the basis for considerable further development. For example, vastly improved microcantilevers photon detectors could be produced by making relatively simple changes in the materials and geometries used in microcantilever fabrication. It is possible to design microcantilevers with much smaller force constants by varying the geometry of the microcantilever, and in contrast to the devices used in this study, mirocantilevers with force constants as small as $0.008 \mathrm{~N} / \mathrm{m}$ can be produced. Since the fundamental mechanical resonance frequency of a microcantilever is proportional to the square root of the spring constant, $k^{1 / 2}$, reductions in force constant can be used to bring resonance into ranges compatible with mechanical chopping frequencies.

The microcantilever spectral response can be easily tailored through the application of specific antireflective coatings and choice of material for fabrication. This means that microcantilevers can be fabricated using standard semiconductor methods and materials, and as a consequence could be mass produced at very low cost. Hence, two-dimensional cantilever arrays based on the technology described here, could become very attractive for a number of applications due to their inherent simplicity, high sensitivity, and rapid response to optical radiation. While the optical readout method is useful with single element designs, practical implementation of microcantilever arrays may require the use of other readout methods, such as piezoresistance or capacitance. Fortunately, the microcantilever technology's compatibility with a variety of readout methods also affords tremendous flexibility to potential system designers.

\section{ACKNOWLEDGEMENTS}

This work was supported by the Laboratory Director's Research and Development Program of Oak Ridge National Laboratory. Oak Ridge National Laboratory is operated for the U.S. Department of Energy by Lockheed Martin Energy Research Corporation under contract DE-AC05-96OR22464.

\section{REFERENCES}

[1] R. J. Kayes, Optical and Infrared Detectors, vol. 19. Berlin: Springer-Verlag, 1977.

[2] J. L. Miller, Principles of Infrared Technology. New York: Van Nostram Reinhold, 1994.

[3] E. L. Dereniak and G. D. Boreman, Infrared Detectors and Systems. New York: Wiley and Sons, 1996.

[4] A. Rogalski, "New Trends in Infrared Detector Technology," Infrared Phys. Technol. 35, 1 (1994).

[5] C. Hanson, "Uncooled Thermal Imaging at Texas Instruments," Infrared Technology XXI, SPIE 2020, 330 (1993).

[6] R. A. Wood, "Uncooled Thermal Imaging with Monolithic Silicon Focal Planes," Infrared Technology XXI, SPIE 2020, 32 (1993).

[7] P. W. Kruse, "Uncooled IR Focal Plane Arrays," Infrared Technology XXI, SPIE 2552, 556 (1995).

[8] P. G. Datskos, P. I. Oden, T. Thundat, E. A. Wachter, R. J. Warmack, and S. R. Hunter, "Remote Infrared Detection Using Piezoresistive icrocantilevers," Appl. Phys. Lett. 69, 2986 (1996).

[9] E. A. Wachter, T. Thundat, P. G. Datskos, P. I. Oden, S. L. Sharp, and R. J. Warmack, "Remote Optical Detection Using Microcantilevers," Rev. Sci. Instrum. 67, 3434 (1996). 
[10] R. Amantea, C. M. Knoedler, F. P. Pantuso, V. K. Patel, D. J. Sauer, and J. R. Tower, "An Uncooled IR Imager with $5 \mathrm{mK}$ NEDT," presented at SPIE, Orlando, FL, 1997.

[11] D. Sarid, Scanning Force Microscopy. New York: Oxford University Press, 1991.

[12] J. H. Hoh, J. P. Cleveland, J.-P. Prater, and P. K. Hansma, "Quantized Adhesion Detected with Atomic Force Microscope," J. Am. Chem. Soc 114, 4917 (1992).

[13] F. Ohnesorge and G. Binning, "True Atomic Resolution by Atomic Force Microscopy Through Repulsive and Attractive Forces," Science 260, 1451 (1993).

[14] J. Varesi, J. Lai, T. Perazzo, Z. Shi, and A. Majumdar, "Photothermal Measurements at PicoWatt Resolution Using Uncooled Micro-Optomechanical Sensors," Appl. Phys. Lett. 71, 306 (1997).

[15] J.Lai, T.Perazzo, Z. Shi, and A. Majumdar, "Optimization and Performance of High-Resolution Micro-Optomechanical Thermal Sensors," Sensors and Actuators (in press).

[16] J. G. E. Harris, D. D. Awschalom, K. D. Maranowski, and A. C. Gossard, "Fabrication and Characterization of 100-nm Thick GaAs Cantilevers," Rev. Sci. Instrum. 67, 3591 (1996).

[17] T. Thundat, P. I. Oden, P. G. Datskos, G. Y. Chen, and R. J. Warmack, "Microcantilever Sensors," presented at The 16th Warner Brandt Workshop on Charged Particle Penetration Phenomena, Oak Ridge Tennessee, 1996.

[18] R. M. White, "Generation of Elastic Waves by Transient Surface Heating," J. Appl. Phys. 34, 3559 (1963).

[19] H. K. Wickramasinghe, R. C. Bray, V. Jipson, C. F. Quate, and J. R. Salcedo, "Photoacoustics on a Microscopic Scale," Appl. Phys. Lett. 33, 923 (1978).

[20] R. G. Stearns and G. S. Kino, "Effect of Electronic Strain on Photoacoustic Generation of Silicon," Appl. Phys. Lett. 47, 1048 (1985).

[21] A. Rosencwaig and M. White, "Imaging of Dopant Regions in Silicon with Thermal-Wave Electron Microscopy," Appl. Phys. Lett. 38, 165 (1981).

[22] T. Figielski, "Photostriction Effect in Germanium," Phys. Status Solidi 1, 306 (1961).

[23] P. Hess and A. C. Boccara, Photoacoustic, Photothermal and Photochemical Processes at Surfaces and in Thin Films. Berlin: Springer-Verlag, 1989.

[24] P. G. Datskos, S. Rajic, and I. Datskou, "Photo-Induced Stress in Silicon Microcantilevers," Appl. Phys. Lett. (submitted).

[25] F. J. von Preissig, "Applicability of Classical Curvature-Stress Relation for Thin Films on Plate Substrates," J. Appl. Phys. 66, 4262 (1989).

[26] R. P. Feynman, R. B. Leighton, and M. Sands, The Feynman Lectures on Physics, vol. 2, 13th ed. Reading, MA: Addison-Wesley Publishing Company, 1964.

[27] P. J. Shaver, "Bimetal Strip Hydrogen Gas Sensor," Rev. Sci. Instrum. 40, 901 (1969).

[28] P. I. Oden, E.A. Wachter, P.G. Datskos, T. Thundat, and R.J. Warmack, "Optical and Infrared Detection Using Microcantilevers," SPIE - Infrared Technology XXII 2744, 345 (1996).

[29] R. C. Weast, Handbook of Chemistry and Physics, 59th ed. Florida: CRC, 1972. 


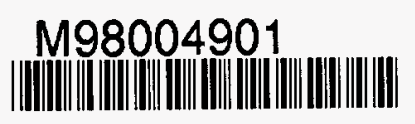

Report Number (14) ORNL/CP-97596

Publ. Date (11)

199804

Sponsor Code (18)

UC Category (19)

$\frac{D O E / M A, X F}{U C-910, D O E / E R}$ 